\title{
SPARSE DFT BASED CHANNEL ESTIMATION IN OFDM SYSTEMS
}

\author{
Maryam K. Abboud ${ }^{1}$, Bayan M. Sabbar ${ }^{2}$ \\ 1,2 College of Information Engineering, Al-Nahrain University, Baghdad, Iraq \\ it18ms@coie.nahrainuniv.edu.iq ${ }^{1}$, bayan.mahdi@ coie-nahrain.edu.iq ${ }^{2}$ \\ Received:17/12/2019, Accepted:7/2/2020
}

\begin{abstract}
Channel estimation is an essential part of Orthogonal Frequency Division Multiplexing (OFDM) communication systems. In this paper, two Discrete Fourier Transform (DFT) improvement algorithms are proposed and compared, where the first one exploits channel sparsity concept while the other considers significant channel coefficients only. In the proposed algorithms; Enhanced and Sparse DFT (E- DFT and S- DFT), different number of significant channel components is selected either by a threshold determining procedure such as in E- DFT, or through determining channel sparsity level such as in S- DFT. In the presence of Doppler frequency shifts, the Inter Symbol Interference (ISI) effect on channel coefficients is successfully reduced using the proposed estimation algorithms. Vehicular A- ITU channel model is considered with a relatively high vehicle speed up to $68 \mathrm{Km} / \mathrm{h}$ in order to test the suitability of the proposed algorithms for mobile systems. E-DFT and S-DFT improves conventional as well as previous suggested works on performance improvement of DFT technique (I- DFT). For 64 subcarriers, S- DFT outperforms E- DFT and I- DFT by about 3dB at a BER of 0.01 with mobility reaches $45 \mathrm{Km} / \mathrm{h}$, and by about $0.4 \mathrm{~dB}$ and $2.5 \mathrm{~dB}$ at a BER of 0.02 with mobility reaches $68 \mathrm{Km} / \mathrm{h}$.
\end{abstract}

keywords: Channel estimation, Sparse channel, SDFT, OFDM.

\section{INTRODUCTION}

In modern communication systems, OFDM is a widely used as a modulation technique due to its ability to overcome the ISI problem in multipath fading environments. OFDM performance mainly depends on the estimation accuracy of channel parameters in the presence of deep fading where pilot aided channel estimation is considered by many estimation techniques [1]- [17]. Pilot aided channel estimation was considered to be the most effective method for Channel Impulse Response (CIR) estimation in which pilots are inserted at different intervals. Different techniques such as Least Squares (LS) and Linear Minimum Mean Square (LMMS) where used. LS estimation method was considered to be the simpler one but it is highly affected by noise [2], [3], [4]. At the other hand, many different algorithms are suggested to suppress the noise in LS and improve its performance by converting the CIR to the time domain. DFT based estimation method proved to have improved performance compared to LS and LMMS in the presence of noise with low implementation complexity [5]. DFT- based channel estimation is a good compromise between performance and complexity, thus its complexity much reduced as compared to LMMS, while it has a performance as good as that of LMMS [6]. In the presence of Doppler shifts, the multipath propagation causes a time variant channel response which required to be recovered correctly. However, due to the sparse structure of scattering objects, other ideas for estimating the CIR with a limited number of pilots had been developed by considering the inherent sparsity of wireless channel in the field of compressed sensing [7]. Channel sparsity means that, the output of Fourier transform for a signal with a small number of non- zero Fourier coefficients, can be sufficiently represented using only $\mathrm{k}$ coefficients [8]. From this standing point, the main contribution of this paper is represented by suggesting and proposing of two algorithms to improve DFT estimation performance. First, an Enhancement DFT method based on a thresholding procedure for significant channel coefficients selection is proposed, while the second depends on the use of the sparse phenomena of the channel to improve the estimation performance of DFT algorithm in 
the presence of Doppler shifts effects. At the other hand, different methods named as Sparse Fourier Transform (SFFT or SDFT), that have been developed in order to reduce the complexity of Fourier transform computations for the sparse signals [9], [10]. Where they didn't used as channel estimation methods, which differs from the proposed one at this point. The rest of this paper is organized as follows; in section II, literature survey was covered the related work. System model with the proposed methodology is figured in section III. The simulation tests and results are presented in section IV. Finally, the main concluding remarks in section $\mathrm{V}$.

\section{Literature ReVieW}

Despite the possibilities of DFT based channel estimation technique which characterized by good performance and simplicity of implementation, several proposals are presented to enhance its performance in fading environments with Doppler effects [6] - [17]. In order to enhance the performance of LS and LMMS, DFT based channel estimation was tested by eliminating noise, the results shows that the Inter Carrier Interference (ICI) problem is reduced, and hence, the performance improved [6]. Conventional and improved DFT methods were proposed and investigated in [11]. Cyclic prefix updated according to computing the maximum energy component within $\mathrm{CP}$ length, where it was chosen according to the index of the maximum energy component and only significant components within the new cyclic prefix are selected while all other channel components are ignored. This gives a better performance compared to conventional DFT method with different Doppler frequencies not exceeding $33 \mathrm{~Hz}$ with a BER of $10^{-3}$. In [12], an iterative and improved DFT based channel estimation method was proposed for MIMO- OFDM system. Only significant channel components within $\mathrm{CP}$ are considered and the other are assumed to be noisy which are set to zero. In [13], an adaptive noise removal was suggested to enhance CIR using transform domain cluster. In [14], a significant channel tap detector (SCTD) algorithm was assumed to predict and ignore the noise power by employing the correlation of two successive preambles, and hence effectively improve the system performance. In order to improve the degraded performance for non- orthogonal channels of multiple antennae, DFT- based channel estimation was investigated in [15] and [16] for OFDM combined with MIMO system, where scheme simulations validate its effectiveness in channel estimation. In [17], two techniques were presented to mitigate the edge effect of conventional DFT method using null subcarriers. By generating virtual carriers at the receiver side and filling the missing pilots, the techniques outperform previously proposed DFT techniques in terms of BER and mean square error (MSE). In [18], an estimate of noise variance from reference signals on multiple OFDM symbols was used to clean the reference signal in the time domain before applying interpolation. A new DFT improvement algorithm based channel estimation was proposed in [19] using guard band prediction with virtual LMMSE interpolation in order to reduce the edge effect and to overcome the problem of pilot mismatching. The traditional DFT method was improved by about $2 \mathrm{~dB}$ gain in SNR at a BER of $10^{-2}$ where it tested in a multipath environment with $100 \mathrm{~Hz}$ Doppler frequency. A new threshold setting methods were proposed in [20] with DFT based channel estimation to eliminate the reference signal from noise. By using a comparison of the total channel energy and the energy of a selected significant channel tabs, Mean Square Error (MSE) was significantly reduced as compared to the conventional methods. In [21], an adaptive channel taps selection was proposed. These taps are detected based on a predetermined threshold while other taps are eliminated.

https://ijict.edu.iq 


\section{METHODOLOGY}

\section{A. System modeling}

The OFDM system of Fig. 1 was modeled and simulated using MatLab simulator in steps representing the main building blocks of the system. After defining of all OFDM system parameters, a QPSK mapping process is implemented first to map a random data previously generated which then converted from serial to parallel flow of data. A number of pilots were determined and inserted within the OFDM symbol at equally spaced locations. The resultant OFDM symbol with pilot subcarriers and data subcarriers are then fed into IFFT process. A CP is added in order to mitigate the Inter Symbol Interference (ISI) problem and maintain orthognality of OFDM symbol [3]. At the receiver side, FFT process was applied to the received OFDM symbol after removing CP. A predetermined channel estimation process was applied to the received data in order to predict the CIR at pilot locations. The estimated channel coefficients are interpolated using linear/ spline interpolation method [22], [23]. In addition to the conventional LS and DFT estimation methods, different estimation techniques where applied in order to enhance the estimation performance in the presence of Doppler effects.

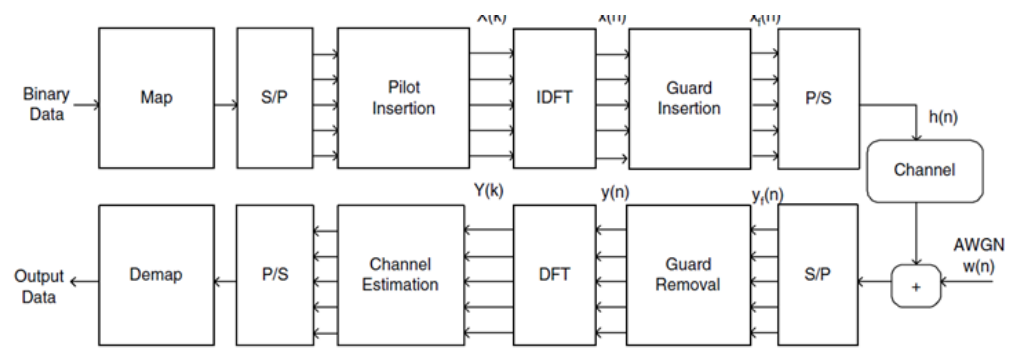

Figure 1: OFDM system [2]

\section{B. Conventional DFT}

In conventional DFT, the estimated channel coefficients using LS algorithm are DFT interpolated. These coefficients transformed into time domain for further processing as shown in Fig. 2. Since CP was chosen to cover the maximum channel delay spread, the noise components of the output of IFFT process outside the CP length are ignored and only the channel components within the CP length are considered. Finally, the considered channel components are DFT interpolated with $\mathrm{N}$ points FFT process, where the result represents the estimated CIR which will be used for signal detection [6], [16].

\section{Enhanced DFT (E- DFT)}

Different approaches proposed by a number of researchers [4], [18], [12] in order to improve the performance of conventional DFT method. The most popular approach assumes that not all channel coefficients (result from conventional DFT estimation) within CP length are significant. The coefficients with maximum power or agreed to be significant with a predetermined threshold are selected, while the others are neglected and considered to be noisy channel coefficients. Finally, the resultant selected coefficient will be used and FFT interpolation applied to re- compute CIR in frequency domain [18], [21]. In this paper, an enhanced approach is proposed in order to improve the performance of the conventional as well as the 
previously improved DFT approaches. The main computation steps of the proposed algorithm are listed below supported by system model of Fig. 3. This algorithm considers all significant channel components whether they are within the CP length or not. In the proposed algorithm:

- The estimated CIR with LS method is fed to $\mathrm{N}$ point IFFT interpolation to derive $h_{I D F T}$.

$$
h_{I D F T}=\operatorname{IFFT}\left(H_{L S}\right)
$$

$H_{L S}$ : channel response after LS estimation, and $h_{I D F T}$ : Time domain channel response.

- The output is divided into two parts, the first part contains CIR along CP length, and the second part represents the reminder CIR outside CP length. For each part, the energy of each channel coefficient is computed and the maximum energy selected. Thus, the maximum energy inside $\mathrm{CP}$ is computed as:

$$
E_{M A X-i n}=\operatorname{MAX}\left(\left|h_{I D F T_{i}}\right|^{2}\right) i=1,2,3, \ldots ., c p
$$

And the maximum energy outside CP length is computed as:

$$
E_{M A X-o u t}=M A X\left(\left|h_{I D F T_{i i}}\right|^{2}\right) i i=c p+1, \ldots ., N
$$

CP: cyclic prefix length.

$E_{M A X-i n}$ : Maximum energy within CP.

$E_{M A X-o u t}$ : Maximum energy outside CP length.

$\mathrm{N}$ : Number of OFDM subcarriers.

- The selected maximum energies are compared with each other and the maximum one determined. The index of the maximum power selected is considered as an updated of effective channel length (L) while the previous one ignored.

$$
L=\operatorname{index}\left(\operatorname{MAX}\left(E_{M A X_{\text {out }}}, E_{M A X_{i n}}\right)\right)
$$

- All CIR coefficients within L are considered while the others are assumed to be noisy components.

$$
h_{I D F T}=\left\{\begin{array}{cc}
h_{I D F T_{i}} & i=1: L \\
0 & \text { otherwise }
\end{array}\right\}
$$

- Finally, N point FFT process is applied to the selected time domain components in order to compute the frequency domain components.

$$
H_{E-D F T}=F F T\left(h_{I D F T}, N\right)
$$

$H_{E-D F T}$ : Enhanced DFT channel response in frequency domain.

\section{DFT Based Sparse Channel Estimation (S-DFT)}

Another approach considers the sparse nature of physical wireless multipath channel is proposed in order to improve the performance of the previously suggested enhanced DFT algorithm. In this approach, only k channel components are selected according to their energies without taking a full advantage of prior channel information such as maximum channel 
delay. The corresponding locations of the selected components are determined. As compared to E- DFT algorithm, there is no matter whether the selected $\mathrm{k}$ coefficients are randomly distributed along the channel length or not, while with E-DFT, a series of channel coefficients within the effective channel length $\mathrm{L}$ are selected and all others outside are ignored. The main computation steps of the proposed approach are listed below with the assistant of Fig. 4.

- The energy of each channel coefficient of $h_{I D F T}$ is computed and k coefficients with maximum energy will be selected. Thus:

$$
E_{M A X_{k}}=M A X_{k \text { components }}\left(\left|h_{I D F T_{i}}\right|^{2}\right) \forall(k \in N), \text { where, } i=1: N
$$

- All k components of the corresponding $E_{M A X_{k}}$ with their indices are selected to compute the CIR of the channel.

$$
h_{I D F T}=\left\{\begin{array}{cc}
h_{I D F T}\left(E_{M A X_{k_{j}}}\right) & j=\text { indices of determined } k \text { components } \\
0 & \text { otherwise }
\end{array}\right\}
$$

- Finally, N point FFT interpolated channel coefficients resultant and used for channel compensation.

\section{Simulation and Performance Test}

The OFDM system was simulated and performance test in the form of BER was carried. Different estimation techniques such as LS, DFT, and I -DFT are evaluated in a fading environment in order to compare their performances with the proposed estimation algorithms; Enhanced DFT ( E-DFT) and Sparse DFT ( S- DFT). Different channel conditions are considered and characterized by A- ITU vehicular channel model of Table I for LTE specifications of mobile systems with six taps. Table II shows the OFDM system parameters considered in simulations according to IEEE 802.11a specifications.

TABLE I

\begin{tabular}{|c|c|c|}
\hline \multirow{2}{*}{ Taps no. } & \multicolumn{2}{|c|}{ Outdoor (vehicular A) } \\
\hline & Relative delay (ns) & Average Power (dB) \\
\hline 1 & 0 & 0 \\
\hline 2 & 310 & -1.5 \\
\hline 3 & 710 & -9.0 \\
\hline 4 & 1090 & -10.0 \\
\hline 5 & 1730 & -15.0 \\
\hline 6 & 2510 & -20.0 \\
\hline
\end{tabular}

Vehicular A Channel Model [24]

TABLE II SYSTEM PARAMETERS

\begin{tabular}{|c|c|}
\hline Parameter & Value \\
\hline Number of transmitted bits & 128000 and 256000 \\
\hline Modulation & BPSK \\
\hline Sampling time(Ts) & $10^{-} 5 \mathrm{sec}$ \\
\hline OFDM subcarriers & 64,128 \\
\hline Number of pilots & 16 \\
\hline Cyclic prefix length (Lcp) & 16,32 \\
\hline Carrier frequency (fc) & $2.4 \mathrm{GHz}$ \\
\hline Doppler frequency shift (fd) $\mathrm{Hz}$ & $30,100,150$ \\
\hline mobile velocity (v) Km/h & $\frac{f d * c}{f c}$ \\
\hline Speed of light (c) $\mathrm{m} / \mathrm{s}$ & $3 \times 10^{8}$ \\
\hline
\end{tabular}

Traditional LS estimation technique with DFT and I- DFT performances are shown in Fig. 5 and 6 in order to compare their performance with the proposed E- DFT and S- DFT algorithms. Different Doppler frequency shifts with 64 and 128 
subcarriers are considered in this simulation for channel estimation with mobility situations. For $f_{d}=30 \mathrm{~Hz}$ with a BER of 0.005 , in Fig. 5, I- DFT outperforms E- DFT by about $0.2 \mathrm{~dB}$, and, S- DFT improves E- DFT and I- DFT by about 0.6 $\mathrm{dB}$ and $0.4 \mathrm{~dB}$, while in Fig. 6, S- DFT improves E- DFT and I- DFT by about $0.9 \mathrm{~dB}$ respectively. As $f_{d}$ increased from $100 \mathrm{~Hz}$ to $150 \mathrm{~Hz}$, the performance of conventional techniques degraded and the improvement of the proposed estimation methods is figured out. Where, for 64 subcarriers, E- DFT improves I- DFT performance by about $2 \mathrm{~dB}$, while S- DFT outperforms E- DFT and I- DFT by about $0.5 \mathrm{~dB}$ and $3 \mathrm{~dB}$ respectively at a BER of 0.02 . At the other hand, for 128 subcarriers, E- DFT outperforms I- DFT by about $1.6 \mathrm{~dB}$, while S- DFT improves E- DFT and I- DFT by about $1.8 \mathrm{~dB}$ and $3.2 \mathrm{~dB}$ respectively at a BER of 0.07 . S- DFT technique was tested considering different number of $\mathrm{k}$ channel components in Fig. 7 in order to compare their performance when channel sparsity degree varying. For $100 \mathrm{~Hz}$ Doppler frequency, as $\mathrm{k}$ increased, S-DFT performance degraded to LS performance, and hence, it can be concluded that the proposed SDFT estimation method works probably assuming that the channel is sparse in nature, which means that most of channel components are highly affected with noise. At the other hand, E- DFT approves its suitability for channel estimation in a multipath environments and its performance outperforms I- DFT. Finally, both E- DFT and S- DFT can be applied for channel estimation of mobile systems in multipath environments with low to moderate Doppler shifts.

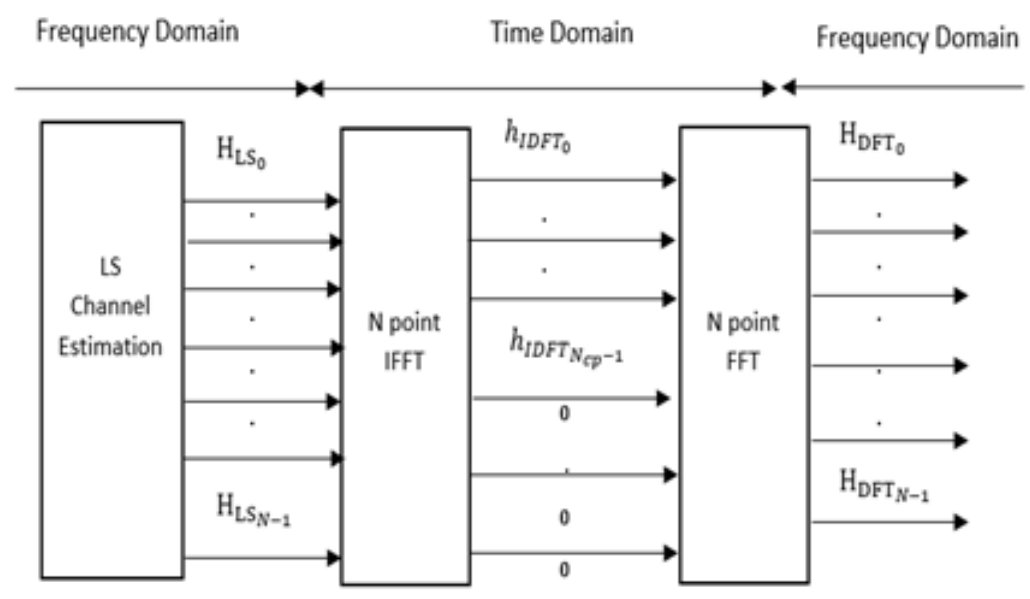

Figure 2: Conventional DFT [5] 


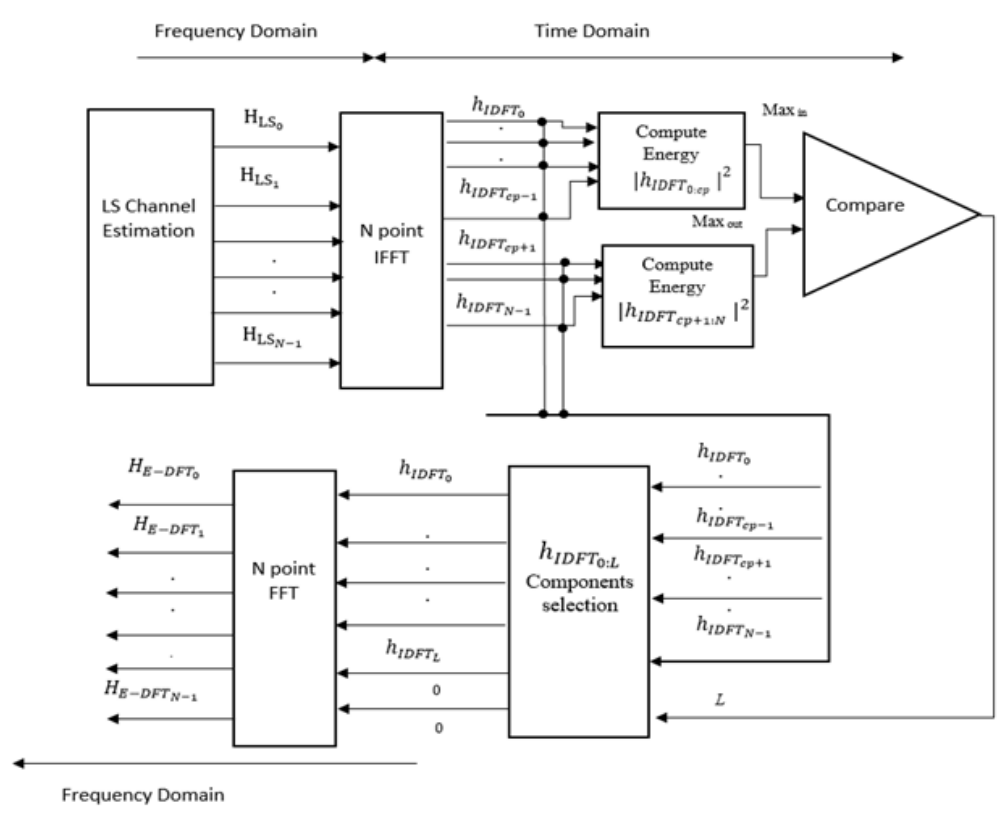

Figure 3: Enhanced DFT

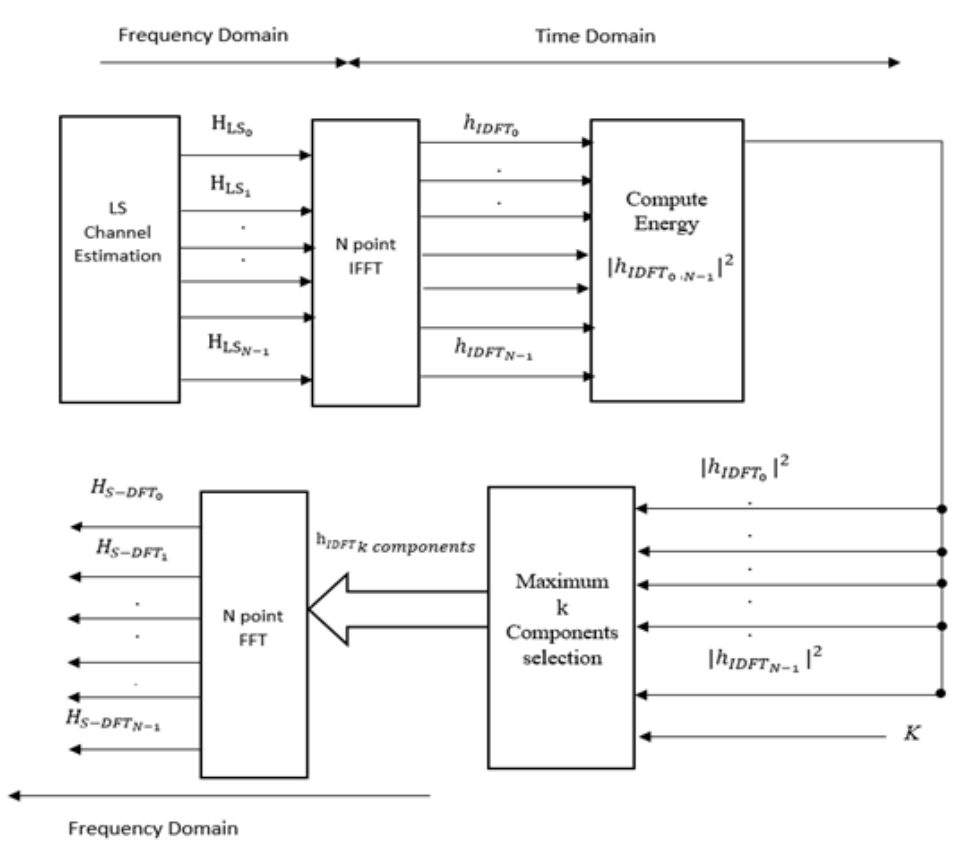

Figure 4: Sparse DFT 

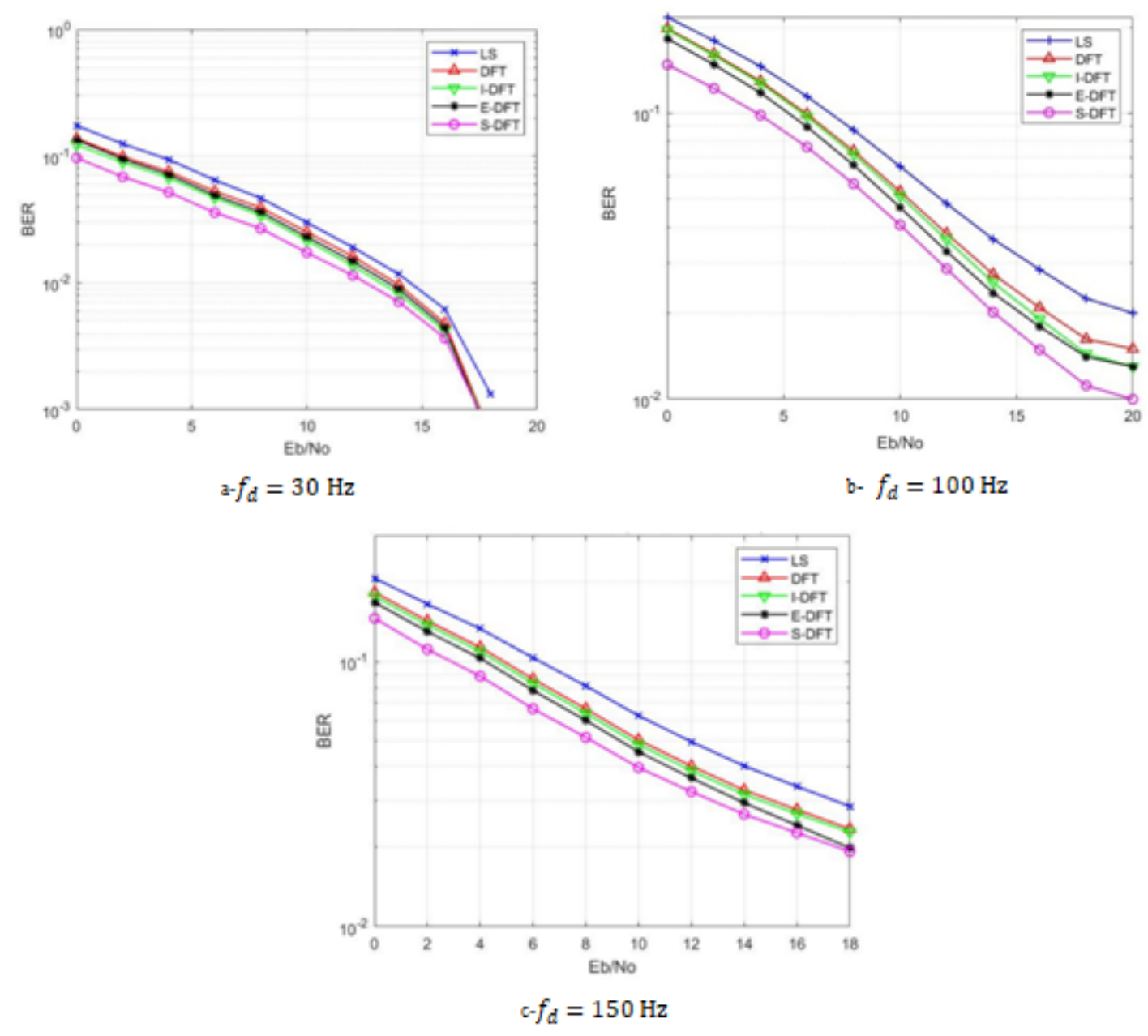

Figure 5: BER performance of $\mathrm{N}=64, \mathrm{~Np}=16, \mathrm{k}=1$ 

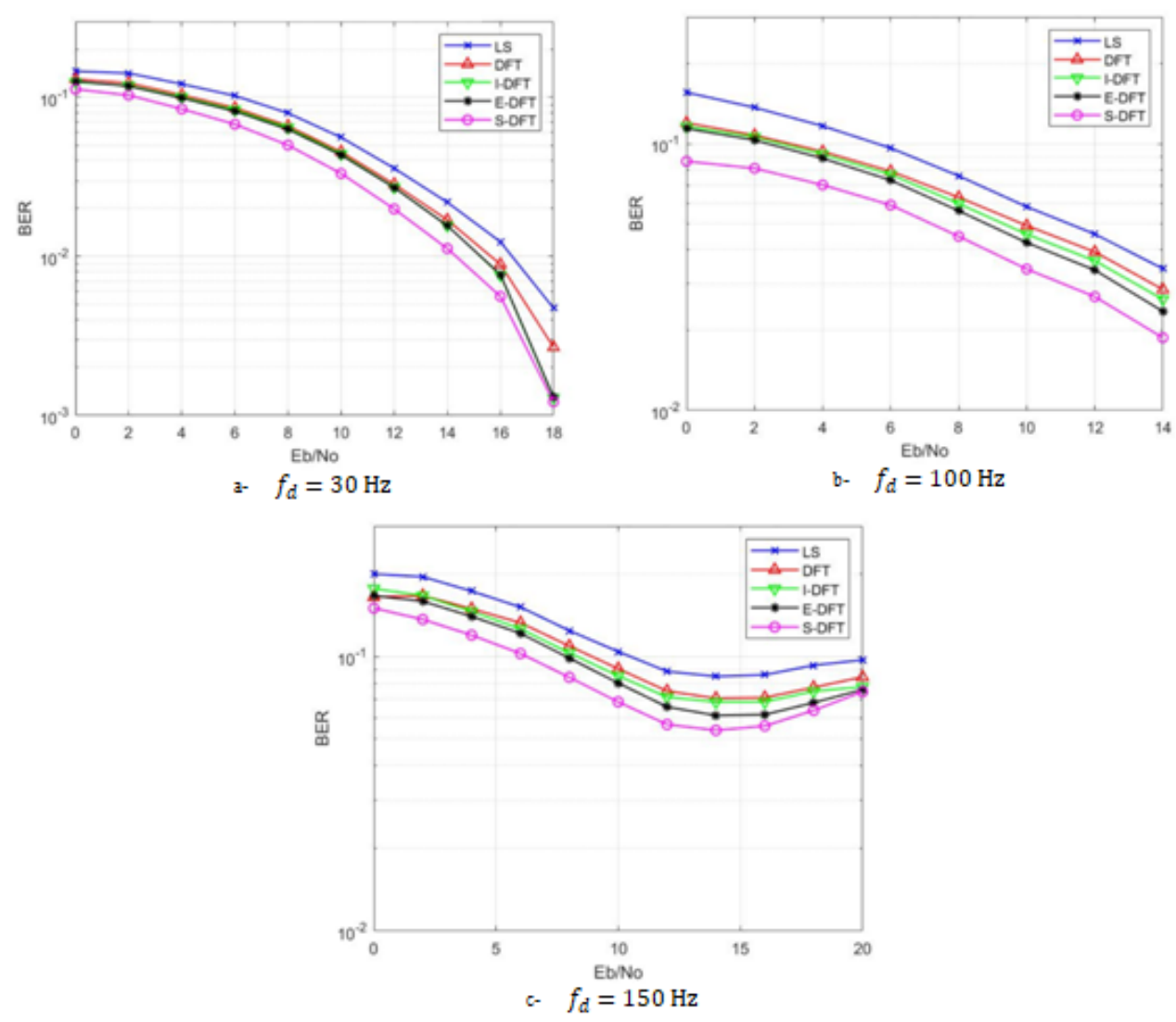

Figure 6: BER performance of $\mathrm{N}=128, \mathrm{~Np}=16, \mathrm{k}=1$
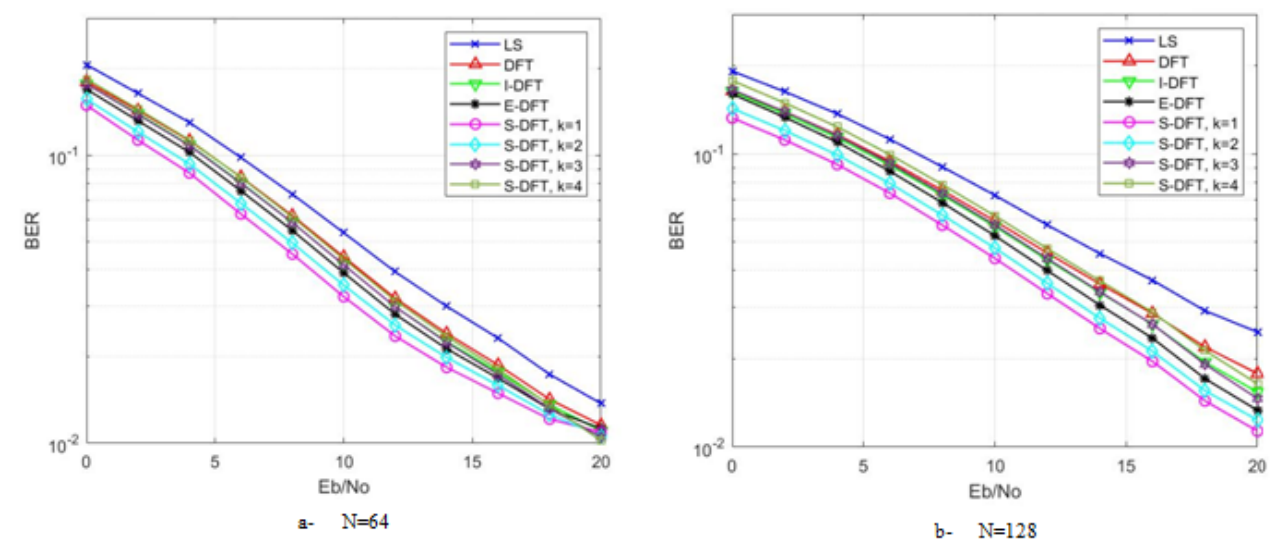

Figure 7: BER performance of $f_{d}=100 \mathrm{~Hz}, \mathrm{~Np}=16$ 


\section{Conclusions}

Pilot aided channel estimation techniques act as the heart of OFDM system since it suffers from changing the channel statistics over time in fading environments. In the proposed work, two channel estimation algorithms are suggested and their performance were tested and evaluated over a multipath fading channel with six taps, were simulation tests compared to different estimation techniques in order to measure their effectiveness in such environment. By applying the system parameters of Table II, the tests shows that the proposed algorithms; E- DFT and S- DFT improves conventional DFT and I- DFT algorithms by about $3 \mathrm{~dB}$ at a BER of 0.01 with a mobility reaches $68 \mathrm{Km} / \mathrm{h}$ for $150 \mathrm{~Hz}$ Doppler frequency shift.

\section{REFERENCES}

[1] K. Zheng, J. Su and W. Wang, " DFT- Based Channel Estimation in COMB- TYPE Pilot- Aided OFDM Systems with Virtual Carrier" , IEEE 18th International Symposium on Personal, Indoor and Mobile Radio Communication, pp. 1 - 5, Sep. , 2007.

[2] S. Coleri, M. Ergen, A. Puri and A. Bahai, " A Study of Channel Estimation in OFDM Systems", IEEE 56th Vehicular Technology Conference ( VTC), Vol. 56, No. 2, pp. 894- 898, sep. ,2002.

[3] S. Wilson, J. Beek, O. Edfors and P. Borjesson, " On Channel Estimation in OFDM Systems", IEEE 45th Vehicular Technology Conference, pp. 815- 819, Jul., 1995.

[4] S. M. Patil, P. A. N Jadhav, " Channel Estimation Using LS and MMSE Estimators" , International Journal on Recent and Innovation Trends in Computing and Communication, Vol. 2, Issue. 3, IJRITCC, March, 2014.

[5] J. Kim, J. Moon, Y. Bang and H. Lee, " A Practical Method of Designing DFT- based Channel Estimator" , IEEE 8th International Conference on Ubiquitous and Future Work (ICUFN), pp. 710- 714, Aug. , 2016.

[6] E. Singh, " A DFT based channel estimation technique in orthogonal- frequency division- multiplexing ( OFDM): A Review" , International Journal of Recent Research Aspects ISSN, Vol. 3, Issue. 1, March, 2016.

[7] P. Pakrooh, A. Amini, F. Marvasti, " OFDM pilot allocation for sparse channel estimation", Springer open EURASIP Journal on Advances in Signal Processing 2012.

[8] Q. Feng, X. Xia, Z. Ye, N. Zhang, " Sparse Multipath Channel Estimation and Decoding for Broadband Vector OFDM Systems" , Mathematics, Computer Science ArXiv 2015.

[9] I. Tariq, M. Qiao, " A fast DFT method for generally k sparse signals recovery", SN Applied Sciences, 2019, ( research article).

[10] Hassanieh H, Indyk P, Katabi D, Price E ( 2012), " Simple and practical algorithm for sparse fourier transform In: Proceedings of the 23rd annual ACM- SIAM symposium on Discrete Algorithms" , pp. 1183- 1194.

[11] N. A. Alazeez and A. A. Kadhim, " Improved DFT- Based Channel Estimation Techniques for OFDM Signal" , International Journal of Engineering Research, Volume No. 5, Issue No. 12, December 2016.

[12] J. Haifang, S. Yin, " An Efficient Iterative DFT- Based Channel Estimation for MIMO- OFDM Systems on Multipath Channels" , Third International Conference on Communications and Networking in China, IEEE, 2008.

[13] H. Zhu, Y. Ge and X. Chen, " DFT- based Adaptive Channel Estimation for OFDM Systems" , IEEE 16th International Conference on Communication Technology ( ICCT), pp. 515- 517, Oct. ,2015.

[14] I. Liu, S. Yang, J. Lin, " Modified DFT- based channel estimation for TD- OFDM communication systems" , IEEE, International Symposium on Intelligent Signal Processing and Communication Systems, Japan, 2013.

[15] Q. Zhang, X. Zhu, T. Yang, and J. Liu, " An enhanced DFT- based channel estimator for LTE- A uplink" , IEEE Trans. Veh. Technol. , vol. 62, no. 9 , pp. $4690-4696$, Nov. 2013.

[16] R. Kaur, C. Kaur, " Discrete Fourier Transform based Channel Estimation Scheme for MIMO- OFDM Communication System" , International Journal of Engineering Research and Applications, Vol. 2, Issue. 3, May- Jun 2012.

[17] L. Zhang, Z. Hong, L. Thibault, " Improved DFT- Based Channel Estimation for OFDM Systems with Null Subcarriers" , IEEE 70th Vehicular Technology Conference Fall, 2009, USA.

[18] H. Yu and C. Yang, " An Improved DFT- based Channel Estimation for Mobile Communication Systems" , Information Theory ( cs. IT), 2015.

[19] J. Seo, J. Wee, et al. , " An Enhanced DFT- Based Channel Estimation Using Virtual Interpolation with Guard Bands Prediction for OFDM" , IEEE International Symposium on Personal, Indoor and Mobile Radio Communications, 2006.

[20] J. Wang, Y. Qiu, " A new DFT based Channel Estimation Method for OFDM Systems over Multipath Channels" , National Conference on Information Technology and Computer Science, 2012.

[21] B. A. Kumar and M. A. Kumar, " Efficient DFT- based channel estimation for OFDM Systems on multipath channels" , International Journal of Conceptions on Computing and Information Technology, Vol. 5, Issue. 1, July, 2017.

[22] T. Hwang and C. Yang, " OFDM and Its Wireless Applications: A Survey", IEEE Transaction on Vehicular Technology, VOL. 58, No.4, pp. 16731694, May, 2009.

[23] A. Sumathi, K. M. Ayesha, S. Kaja Mohideen, " An Improved Method of MIMO- OFDM Channel Estimation Using Chaotic Pilot Sequence" , ARPN Journal of Engineering and Applied Sciences, VOL. 10, NO. 8, 2015, pages 3544- 3549.

[24] S. Ahmadi, R. Srinivasan, H. Choi, J. Park, J. Cho and D. Park, " Channel Models for IEEE 802. 16m Evaluation Methodology Document" , IEEE C802.16, Mar, 2007. 